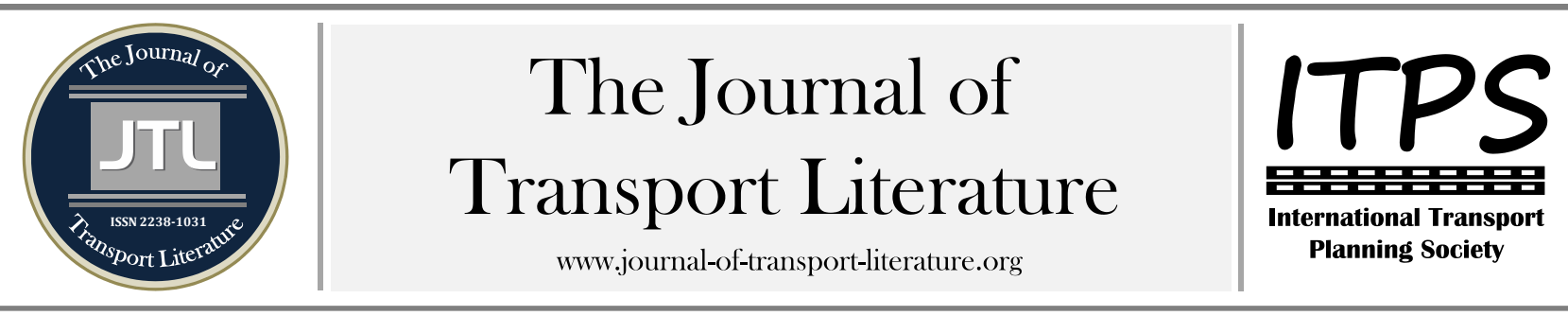

\title{
Análisis del movimiento de pasajeros en la ruta 447 Morón-Ciego de Ávila
}

\author{
Lorenzo Dominico Díaz'; Nicolas Quintana Bernabé \\ Universidad de Ciego de Avila, Ciego de Avila, Cuba
}

\section{Article Info}

Palabras clave:

transportación de pasajeros demanda

rutas

indicadores

anova

Submitted 21 Mar 2014

received in revised form 19 Sep 201 accepted 9 Oct 2014.

Licensed under

Creative Commons

CC-BY 3.0 BR.

\section{Resumen}

El diseño del flujo de transportación mediante el método del perfil de ocupación de los medios de transporte es muy efectivo en estudios de sistemas regulares de transporte de pasajeros, ya que aporta un considerable y abarcador volumen de información sobre estos sistemas, aun así éste método ha sido poco empleado en estudios de transporte intermunicipal en Cuba. En el presente trabajo se realiza un análisis del movimiento de pasajeros en la Ruta 447 Morón-Ciego de Avila a partir de un muestreo de las cantidades de personas traslados y del cálculo de los indicadores técnicos del trabajo de estos medios. Para la recopilación de la información fueron encuestadas cincuenta y seis vueltas en los ocho turnos que realizan los medios cada uno de los siete días de la semana. El análisis por días de la semana mostró que el viernes y el domingo fueron los días de mayor y menor transportación se realizaron respectivamente, mientras que el análisis de los horarios se destaca que la salida de las 3: 00 pm resultó ser la más demandada, así mismo la salida de las 5:30 pm fue en la que menor número de viajeros se transportó, por otra parte las pruebas de Turkey y de Ducan para este conjunto de datos encontraron diferencias significativas de las medias para un nivel del 5\%. Fue encontrada gran irregularidad entre ambos viajes (ida y regreso) lo que puede ser corregido con la utilización de ómnibus de menor capacidad en el turno de la 5:30 pm. De acuerdo a los resultados obtenidos el estudio concluye que para mejorar el servicio que en la actualidad se presta con 4 ómnibus debería ser incrementado con dos ómnibus más y luego reestructurar los horarios de salida teniendo en cuenta las salidas de los otros sistemas de transporte (particular, ferroviario y ómnibus de otros organismos).

+ Corresponding author. Lorenzo Dominico. Universidad de Ciego de Avila. Ciego de Avila CP 4560. Cuba

E-mail address: lorenzodayron@gmail.com.

\section{Introduccion}

El transporte de pasajeros intermunicipal estatal en Cuba tiene como primera prioridad el encargo social, sin embargo muy pocos trabajos se han realizado para evaluar su trabajo con el empleo de herramientas científicos que avalen la toma de decisiones encaminadas a mejorar la eficiencia de este servicio. En el presente trabajo se emplea el método de análisis del perfil de los pasajeros de la Ruta 447 Morón - Ciego de Avila, donde se realizó un profundo análisis estadístico para establecer el comportamiento del movimiento de pasajeros entre los diferentes días de la semana y establecer las variaciones en las cantidades de viajeros en los distintos horarios de un mismo día y entre los diferentes tramos. El recorrido de esta Ruta transcurre por lugares y enlaces de gran significación social, por lo que fue determinado los niveles de saturación de cada uno de los tramos, la distancia media de transportación, los factores que influyen en el bajo valor dela velocidad de explotación. Todos estos aspectos constituyen para los directivos del sector una importante contribución para la planificación y el mejoramiento de este servicio.

El transporte de pasajeros por carreteras, es una rama determinante en el desarrollo del país y asegura como los demás medios del transporte el ritmo de la producción (Cárdenas-Garnier 2009). Para las autoridades suecas, su meta en política de transporte es asegurar un transporte socialmente eficiente y sostenible para sus ciudadanos y para la economía, según consideraciones de Horn y Rude Anna (2014).

Pardo (2008) en su trabajo plantea la tesis que el transporte entre ciudades, constituye uno de los servicios públicos de mayor impacto en la sociedad, por lo que su mejora es crucial para el auge sostenible de ciudades en términos; ambiental, económico, político y en las condiciones de urbanismos.

Para López Olivera (2008) las concentraciones urbanas modernas tienen en el transporte de pasajeros uno de los problemas más difíciles y onerosos de solucionar, pero al mismo tiempo impostergable, ya que en la actualidad el desarrollo de un país depende en mucho del transporte y la modalidad de las personas.

Según Site y Salucci (2009) en la actualidad las empresas dedicadas a la transportación de pasajeros tienen como objetivo ofrecer un servicio eficiente y seguro. Zhou et al (2011) son del criterio que los usuarios del transporte público, buscan un servicio regular, con costos adecuados, alto confort y seguridad.

Para el Grupo de Trabajo de Transporte Público Urbano Colectivo -GTTPUC- (2009), el transporte público por muchas las razones es una actividad vital para la eficiencia y la equidad de las ciudades. Es más eficiente que el transporte motorizado privado en términos pasajeros transportados por unidad de espacio, energía consumida e impacto ambiental y es que la ineficiencia del automóvil se aprecia en tres parámetros: bajo índice de ocupación (aproximadamente 1,3 
pasajeros de media en áreas urbanas y un 25-35\% de la capacidad en los vehículos usuales de 5 plaza), bajo grado de utilización (una hora diaria, un $4 \%$ de su vida útil) y elevado consumo de energía por unidad transportada.

Las empresas transportistas cubanas son poco eficientes, necesitan aprovechar al máximo las moto-recursos disponibles y satisfacer con calidad el servicio de transportación.

Por ello el objetivo del presente trabajo fue diseñar el flujo de transportación de pasajeros de la Ruta 447 Morón-Ciego de Ávila, a partir del análisis del movimiento de pasajeros y el cálculo de los indicadores técnicos durante la transportación.

Para el cumplimiento del objetivo trazado fueron realizadas las siguientes tareas; el estudio del perfil ocupacional en la Ruta 447, el análisis de las características de los recorridos de ida y regreso de la ruta, los niveles de saturación de cada tramo, el cálculo de los indicadores de utilización de los ómnibus a lo largo de la ruta y finalmente el cálculo de la variación del flujo de pasajeros atendiendo a los parámetros de tiempo, tramo y dirección de la ruta.

El trabajo está estructurado como sigue, una introducción donde se exponen resultados de diferentes autores sobre el transporte de pasajeros así como las tareas realizadas para el cumplimiento de los objetivos previsto, una sección donde se describen diferentes metodologías utilizadas por otros autores y la empleada en este trabajo para la recopilación de la información sobre los medios de la Ruta 447 y para el procesamiento estadístico de la información, luego una sección en la que se establecen los índices y coeficientes técnicos del trabajo de los medios así como sus expresiones para el cálculo de los mismos, una sección donde se exponen y analizan los resultados obtenidos y finalmente las conclusiones y recomendaciones del trabajo.

\section{Metodología}

Los estudios de movimiento de pasajeros se deben efectuar cada cinco años para todas las rutas, en Cuba éstos se realizan entre los meses de septiembre a diciembre por ser un período considerado normal en el funcionamiento de la actividad de transportación por ómnibus. Una gran variedad de métodos son empleados para la evaluación de esta actividad.

Moncada y Valente (2005) recomiendan realizar mediciones periódicas a través de indicadores relacionados con la operación global del servicio, mientras que Rocha Murça y Muller (2014) realizaron un estudio econométrico para facilitar la obtención de modelos lo más representativo posible. El análisis de la estructura modal fue empleado por Miralles-Guasch (2012), pues para ellos este análisis constituye una fuente de información básica sobre los desplazamientos en una región dada, desde la perspectiva de la población que se mueve o, lo que es lo mismo, desde la demanda y no desde la oferta de medios de transporte. En cambio para Cantillo (1995) la mejor información sobre los viajes de transporte urbano es la proveniente de encuesta origen-destino.

Por su parte Posada y Gózalez (2010), desarrollaron una metodología para el estudio de la demanda de transporte público rural y para; conocer el número de pasajeros que suben y bajan de un vehículo, los pasajeros movilizados por viaje, las cargas de pasajeros en las zonas, y las zonas de mayor demanda por ascensos y descensos.

En años pasados fueron realizados numerosos estudios para evaluar las redes de transporte público, como el realizado por Soltani, Zargari y Esmaili (2013).

El método de análisis del perfil de pasajeros ha sido usado para conocer el comportamiento de viajeros. Royo Vela (2010) lo empleó para recabar información sobre los perfiles de los viajeros en vuelos de bajo costo y de sus preferencias por las diversas características o atributos de este tipo de vuelo. Para comprender mejor la experiencia de los viajes de los pasajeros de cruceros que visitan por algunas horas el puerto de Cartagena de Indias fue utilizado este método por Brida (2010).

Para analizar el trabajo de la ruta 447 escogimos el método de tabla o perfil, la información fue recogida por inspectores ubicados en cada puerta del ómnibus con un modelo previamente elaborado controlando en cada parada las cantidades de pasajeros que suben, bajan y los que permanecen en el ómnibus.

Fue encuestado el total de los viajes programados durante toda la semana, según los resultados pudimos conocer; el nivel de saturación durante el recorrido, las paradas donde se requieren otros estudios que permitan conocer con mayor detalle el comportamiento del itinerario y el comportamiento de los intervalos de movimiento.

La información obtenida fue revisado y aprobado después y procesada con el uso del paquete estadístico SPSS v20, realizando una ANOVA de un factor para un $\alpha=5 \%$ para establecer la existencia o no de diferencias significativas entre las medias de los tratamientos considerados en el trabajo: los horarios de un mismo día, los mismos horarios de cada día y entre los viajes de ida y de retorno. En los casos de existencia de diferencias significativas se aplicó la prueba de Tukey para conocer el comportamiento de estas diferencias, lo cual nos permitió establecer los diferentes grupos de variables.

En esta ruta prestan servicio un parque de cuatro ómnibus de gran porte; dos remolques (una cuña tractora Internacional y otra Frehitline), dos ómnibus marca Girón articulados.

La longitud a recorrer en ida es de 37,7 km y en regreso es de 37,6 km. También los ómnibus realizan un recorrido cero o en vacío de 1,3 km. Cada ómnibus recorre en su turno de trabajo 150,6 km ocupados y 5,2 km vacíos, recorriendo en el día $155,8 \mathrm{~km}$ y la ruta completa de $620,6 \mathrm{~km}$ diarios. El tiempo de recorrido tanto en la ida como en el retorno es de 70 minutos. Cada día se realizan ocho salidas, tabla 1 . Fueron encuestados 56 vueltas (112 viajes).

Tabla 1 - Itinerario de la ruta 447 Morón-Ciego de Avila

\begin{tabular}{|l|l|l|l|l|l|l|l|l|}
\hline \multicolumn{5}{|l|}{ Horas de salidas } & \\
\hline Ida & $5: 35 \mathrm{am}$ & $7: 30 \mathrm{am}$ & $8: 20 \mathrm{am}$ & $9: 50 \mathrm{am}$ & $11: 30 \mathrm{am}$ & $1: 00 \mathrm{pm}$ & $3: 00 \mathrm{pm}$ & $5: 30 \mathrm{pm}$ \\
\hline Regreso & $7: 20 \mathrm{am}$ & $9: 00 \mathrm{am}$ & $10: 05 \mathrm{am}$ & $11: 20 \mathrm{am}$ & $1: 20 \mathrm{pm}$ & $3: 00 \mathrm{pm}$ & $4: 30 \mathrm{pm}$ & $6: 50 \mathrm{pm}$ \\
\hline
\end{tabular}

Existen 37 paradas en cada sentido de circulación. La distancia promedio entre ellas es de 0,98 km. Respondiendo a su asignación podemos clasificarla dentro de las rutas fundamentales y su itinerario está en coordinación con los horarios de trabajo y de movimientos habituales de la población. 


\section{Indices Técnicos}

Fueron analizados los índices técnicos de operaciones y a partir de los mismos se determinaron las causas que originan la ineficiencia en el proceso de transportación. Raña González et al (2004) refieren que el análisis de estos índices permite conocer las divergencias con las condiciones trazadas de operación, adoptar un plan de acciones para elevar la eficiencia del trabajo de explotación, lo que de hecho influye directamente sobre los costos de transportación y en la satisfacción de los pasajeros.

En nuestro trabajo fueron calculados los siguientes indicadores:

1. Coeficiente de irregularidad por tiempo de la ruta (Kit)

2. Coeficiente de irregularidad por tramo de la ruta (Kitr)

3. Coeficiente de irregularidad por dirección de la ruta (Kid)

4. Distancia promedio a que se transporta un pasajero en la ruta, km (Lprom)

5. Coeficiente de aprovechamiento de la capacidad dinámica ( $\delta$ din)

6. Coeficiente de aprovechamiento de la capacidad estática ( $\delta$ est)

7. Coeficiente de aprovechamiento del recorrido $(\beta)$

8. Coeficiente de no linealidad (CL)

9. Velocidad de Utilización $(\mathrm{Vu})$

10. Cantidad de ómnibus necesarios en la ruta (Vehnec)

\section{Resultados}

\subsection{Análisis estadístico de la información}

El análisis de los días de la semana durante los viajes Morón-Ciego de Avila arrojó que como promedio, el viernes fue el día en que se produjo la mayor transportación, mientras que el domingo resultó ser de menor números de pasajeros que utilizaron este servicio. Al aplicar la prueba de Tukey no existe diferencias entre las cantidades de pasajeros transportados entre los dos sentidos. Para este conjunto de datos todos los variables días fueron agrupadas en un solo subconjunto.

El análisis de los horarios de las salidas desde Morón se obtuvo que de las ocho salidas diarias la de 3:00 pm resultó ser en la que mayor número de pasajeros se transportó, mientras que la de menor ocupación resultó ser la salida de 5:30 pm. Esto se explica porque en el intervalo comprendido entre 2:00 pm a 4:00 pm no hay otras opciones de transporte, en cambio a parir de las 4:00 pm existen varias, como son; salida de un tren con destino a Ciego de Avila y numerosos ómnibus provenientes de otros orígenes hacia el mismo destino. La prueba de Tukey para este conjunto de datos encontró diferencias significativas de las medias para un nivel de 0,05.

Respecto a la información de los viajes de regresos (Ciego de Avila-Morón), según la estadística descriptiva también coinciden el viernes y el domingo como los días de mayor y menor ocupación respectivamente. Para estas variables el ANOVA no reportó diferencias en los valores medios de ocupación entre los días de la semana.

El análisis de los horarios de las salidas en los viajes de retorno muestra que la salida de 7:30 am resultó la de mayor ocupación, se explica por la gran demanda de estudiantes que utilizan esta salida para su traslado hacia la Universidad, mientras que la de menor ocupación resultó ser la salida de 6:50 pm, pues veinte minutos antes existe una salida de un tren con destino a Morón. Para este conjunto de datos tanto la prueba de Tukey encontró diferencias significativas de las medias para un nivel de 0,05 .

\subsection{Resultados de los indicadores del trabajo de los medios}

\subsubsection{Comportamiento del Kit}

El tiempo promedio del total de los viajes estudiados fue de 3920 minutos, la cantidad medio de pasajeros transportados fue de 8826, para un promedio por viaje de 158 pasajeros durante los viajes de ida y de 151 durante el viaje de regreso. Por lo tanto el Kit tuvo un resultado de 55,9 y de 55,8 para viaje de ida y regreso respectivamente.

\subsubsection{Coeficiente de irregularidad por tramo de la ruta (Kitr)}

Para el cálculo de este coeficiente tomamos el volumen máximo de pasajeros del tramo más saturado durante los 112 viajes, que resultó ser el tramo comprendido entre la terminal de Ciego de Avila y la Universidad. El Kitr se comportó a un $123 \%$, lo que nos indica que para este tramo el ómnibus circula sobre saturado, existiendo demanda para otro medio en el horario de salida de 7:20 am.

\subsubsection{Coeficiente de irregularidad por dirección de la ruta (Kid)}

Para calcular este coeficiente tomamos el número máximo de pasajeros en una dirección dada, en este caso viaje de retorno salida 4:30 pm. Ciego de Ávila - Morón del miércoles y el número mínimo de pasajeros transportados en la otra dirección, en este caso viaje de ida salida 5:30 pm. Morón - Ciego de Ávila del lunes. El resultado fue de 6,8 lo que nos indica que existe una gran irregularidad entre ambos viajes lo que se puede corregir con la utilización de un ómnibus de menor capacidad en el viaje de Ciego a Morón.

\subsubsection{Distancia promedio a que se transporta un pasajero en la ruta (Lprom)}

Escogimos para nuestro análisis el viaje de 5:35 am Morón- Ciego de Avila del lunes, en el cual se transportaron 149 pasajeros. Se obtuvo que la distancia promedio a la que se transportó un pasajero es de 33,1 km, lo que indica que independientemente de la hora, en el municipio de Morón existen las condiciones de mercado dada la cantidad de personas que utilizan este servicio para su traslado hasta la capital provincial.

\subsubsection{Coeficiente de aprovechamiento de la capacidad dinámica (ddin)}

El tráfico real fue determinado considerando la cantidad de pasajeros transportados durante los 112 viajes. El resultado obtenido fue de $128 \%$, lo que muestra que los ómnibus transitan con un número de pasajeros superior a sus capacidades, en algunos casos con gran saturación de viajeros. 


\subsubsection{Coeficiente de aprovechamiento de la capacidad estática (Sest)}

El resultado de este coeficiente coincide con el anterior y es que la cantidad de pasajeros transportados es mayor que el tráfico posible a realizar, así como la cantidad de pasajeros posibles a transportar. Esto se debe a la existencia de cuatro tramos (Morón-Villa-UCI-La Loma-Universidad) en que los ómnibus sobrepasan su capacidad nominal, en clara violación de las normas establecidas.

\subsubsection{Coeficiente de aprovechamiento del recorrido $(\beta)$}

La longitud de un viaje redondo es de $37,7 \mathrm{~km}$ en viaje de ida y $37,6 \mathrm{~km}$ en viaje de retorno más $1,3 \mathrm{~km}$ en cada sentido desde la base a la terminal y viceversa. Por lo que el valor del coeficiente de aprovechamiento del recorrido ( $\beta$ ) es de un 97 $\%$, lo que se considera muy bueno para este tipo de servicio.

\subsubsection{Coeficiente de no Linealidad (CL)}

Este es un coeficiente tuvo un comportamiento de $88 \%$, el cual puede ser considerado como alto, indicando que la ruta cumple las condiciones que anteriormente habíamos señalado y atendiendo a la ubicación geográfica de estos dos municipios dentro del territorio de la provincia.

\subsubsection{Velocidad de utilización ( $\mathrm{Vu}$ )}

La velocidad de utilización calculada fue de $32 \mathrm{~km} / \mathrm{h}$, valor bajo influenciado por la corta distancia (una media de 0,98 $\mathrm{km}$ ) entre las paradas 37 oficiales que tiene que realizar el ómnibus. También influye el tiempo en las paradas para la subida y bajada de los pasajeros, sobre todo en aquellos ómnibus que poseen solo una puerta para las dos acciones, lo que ocasiona una considerable pérdida de tiempo, como lo reportó Fernández y Tyler (2005).

\subsubsection{Cantidad de ómnibus necesarios en la ruta (Vehnec)}

Según los cálculos realizados para satisfacer la demanda sin exceder utilización de las capacidades estática de los ómnibus, se necesitarían 6 ómnibus, en la actualidad el servicio se presta con 4 ómnibus lo que conllevaría a una reestructuración de los horarios de salida lo que mejoraría considerablemente el servicio y la satisfacción de los usuarios. Sería muy conveniente planificar dos salidas nuevas, una a las 7:00 am y otra a las 2:00 pm, ambas como servicio expreso, con menor número de paradas lo que disminuirían el tiempo de viaje.

\section{Conclusiones}

En el presente estudio fue realizado un análisis del movimiento de los viajeros transportado por la Ruta 447 MorónCiego de Avila, en el mismo se apreció que el método de tabla o perfil de ocupación resultó muy apropiado para evaluar el resultado del trabajo de transportación de pasajeros ejecutado por la Ruta 447 Morón-Ciego de Avila. Los resultados del análisis estadístico de la información recopilada sobre el número de pasajeros transportados mostraron la no existencia de diferencias entre las transportaciones en los dos sentidos, en ambos casos el viernes fue el día de mayor transportación. Quedó demostrado que la salida de las 3:00 pm es la más demanda por los usuarios entonces la Empresa debe garantizar para esta salida los ómnibus de mayor capacidad

Respecto al comportamiento de los indicadores del trabajo de los medios se aprecia correspondencia de éstos con la clasificación y el encargo social de la Ruta 447, lo que se aprecia en el número de paradas y las distancias entre éstas, con una distancia media entre paradas de $1,01 \mathrm{~km}$, una magnitud muy pequeña lo que trae como consecuencias un elevado tiempo de inmovilización de los medios y el consiguiente aumento de consumo de combustible. Aun cuando en este servicio es prioritario el encargo social sería conveniente realizar una reubicación de las paradas para disminuir estos tiempos.

Los resultados obtenidos demuestran la existencia de una demanda de pasajeros real que garantiza la ocupación de la Ruta 447 y que aporta beneficios económicos, pero a su vez debe ser controlado el número de pasajeros según las capacidades de los medios. Dada la existencia de demanda se precisa de un aumento del número de salida estableciendo de servicios expresos, que no es común en Cuba, por lo cual es recomendable estudiar los horarios, las paradas y los precios para este nuevo servicio. Así debería realizarse estudios en los meses de junio-agosto donde todo el sistema de educación y muchos trabajadores están en disfrutando de sus vacaciones por lo que aumenta considerablemente la movilidad de personas.

\section{Referencias}

Brida, J. (2010) Análisis del perfil y satisfacción de los pasajeros de cruceros que visitan el puerto de Cartagena de Indias. Revista de investigación en turismo y desarrollo local, vol. 3, n. 8, pp. 16.

Cantillo, V. (1995) Propuesta de una nueva metodología para la planificación del transporte urbano. Revista Ingeniería \& Desarrollo. Universidad del Norte, n. 1, pp. 59-72.

Cárdenas-Garnier, M. A. (2009) Evaluación de los parámetros del clima en el interior de ómnibus de largas distancias. Ingeniería Mecánica, vol. 12. n.1, pp. 13-20.

Departamento Administrativo Nacional de Estadística - DANE - (2009) Metodología encuesta de transporte urbano de pasajeros. DANE, n, 55. pp, 45.

Fernández, R. y Tyler, N. (2005). Effect of passenger/Bus/Traffic interactions on bus stop operations. Transportation Planning and Technology, n.4, pp. 273-292.

GTTPUC. (2009) Factores determinantes del transporte público urbano colectivo en España. Federación Española de Municipios y Provincias, pp. 1-45.

Horn, V. R, y Rude, A. (2014) Peak-load pricing in public transport: a case study of Stockholm. Journal of Transport Literature, vol. 8, n. 1, pp. 52-94.

López Olivera, M. A. (2013) El transporte de pasajeros y el sistema vial en la ciudad de México. Texto no publicado. Disponible en www.juridicas.unam.mx. Accedido en:15 de noviembre de 2013.

Miralles-Guasch, Carme. (2012) Las encuestas de movilidad y los referentes ambientales de los transportes. EURE, vol 38, n. 115 , pp. 33-45.

Moncada Arsitobal, C. A y Burbanano Valente, J. (2005) Modelo de estructura empresarial para el transporte público colectivo. Universitas Sychologica, Pontifica Universidad Javeriana. Bogotá, Colombia, vol. 4, n. 003, pp. 1-25.

Pardo, F. (2008) Developments in public-transport systems in the cities of Latin America. Economic Commission for Latin America and the Caribbean (ECLAC). Bulletin FAL, n. 25, pp. 35. 
Posada Henao, J. y González Calderón, C. A. (2010) Metodología para estudio de demanda de transporte público de pasajeros en zonas rurales. Revista de la Facultad de Ingeniería. Universidad de Antioquia, n. 53 pp. 106-118.

Raña González, L. del A., Castillo Asencio, O. y Rodríguez Ramos, P. (2004) Cómo elevar la eficiencia en la selección de vehículos. Ingeniería Mecánica, n. 1, pp. 37-43.

Realidades y perspectivas del transporte de pasajeros en Cuba.

Rocha Murça, M. y Müller, C. (2014) Transporte coletivo urbano: uma análise de demanda para a cidade de Salvador. Journal of Transport Literature, vol. 8, n. 1, pp. 265-284.

Royo Vela, M. y Martínez García Esther. (2010) Un análisis de segmentación y perfil de los segmentos de los usuarios de vuelos de bajo coste. Cuadernos de Turismo, n. 26, pp. 303-306.

Site, P. y Salucci, M. (2009) Efficiency in sustainable mobility. European Commission DG Energy and Transport. Thematic Research Summary. Transport Research Knowledge Centre.

Soltani, A; Zargari Marandi, E. y Esmaili Ivaki, Y. (2013) Bus route evaluation using a two-stage hybrid model of Fuzzy AHP and TOPSIS. Journal of Transport Literature, vol. 7, n. 3, pp. 34-58.

Zhou, Y., Thill, J. y Huang, Z. (2011) Design of a user centric decision support tool for fixed-route bus travel planning. Applied Geography, n. 31, pp.1173-1184.

\section{Abstract}

This work aimed to design the flow of passenger transportation 447-Morón-Ciego de Ávila, Cuba, from the calculation of the technical indicators and the number of means to satisfy the needs of passenger transportation route. The length of the course during the first leg is $37.7 \mathrm{~km}$ and for the return of $37.6 \mathrm{~km}$, with the total length of around $75.3 \mathrm{~km}$. Were surveyed 56 laps in eight innings, allowing us to analyze the profile of occupation of this route on their trips to and back, thereby it was determined, the level of use of the buses, the degree saturation different sections, and the variation in response to passenger flow parameters: time span, the average distance and direction to a passenger is carried on the route.

Key words: transportation of passengers, demand, routes, indicators, anova. 